\title{
Fast explicit nonlinear model predictive control via multiresolution function approximation with guaranteed stability
}

\author{
Sean Summers, Davide M. Raimondo, Colin N. Jones, \\ John Lygeros, Manfred Morari \\ Automatic Control Laboratory, ETH Zürich, Switzerland \\ Email: \{summers, davide.raimondo, cjones, lygeros, \\ morari\}@control.ee.ethz.ch
}

\begin{abstract}
In this paper an algorithm for nonlinear explicit model predictive control is introduced based on multiresolution function approximation that returns a low complexity approximate receding horizon control law built on a hierarchy of second order interpolets. Feasibility and stability guarantees for the approximate control law are given using reachability analysis, where interval methods are used to construct a capture basin (feasible region). A constructive algorithm is provided that combines adaptive function approximation with interval methods to build a receding horizon control law that is suboptimal, yet with a region of guaranteed feasibility and stability. The resulting control law is built on a grid hierarchy that is fast to evaluate in real-time systems.
\end{abstract}

\section{INTRODUCTION}

The implementation of a receding horizon model predictive control (MPC) (Morari and Lee [1999], Magni et al. [2009], Rawlings and Mayne [2009]) law requires the solution of an optimization problem online at each sampling instant. It has become well-known that this optimization problem can be posed parametrically, with the measured state $x$ as the parameter

$$
J^{*}(x):=\min \{h(x, u) \mid g(x, u) \leq 0\} .
$$

The offline computation of this parametric problem results in an explicit optimal control law $u^{*}(x)$ mapping the current state $x$ to the optimal system input Bemporad et al. [2002], Johansen and Petersen [2000], M. M. Seron and Dona [2000].

Methods for the approximation of explicit solutions of nonlinear model predictive control (NMPC) problems have been addressed recently by various authors (e.g. see Canale et al. [2009a], Johansen [2004]). In Canale et al. [2009a], the authors compute an approximate control law $\tilde{u}(x)$ with a bound on the controller approximation error $\left(u^{*}(x)-\right.$ $\tilde{u}(x)$ ), from which performance and stability properties are derived using Set Membership (SM) function approximation theory. In Johansen [2004], the authors use multiparametric nonlinear programming to compute an explicit approximate solution of the NMPC problem defined on an orthogonal structure of the state space partition. An additional example of the approximation of explicit solutions of NMPC can be found in Pin et al. [2009].

\footnotetext{
* This research was partially supported by the Swiss National Science Foundation under grant 200021-122072 and by the European Commission under the project Feednetback FP7-ICT-223866 (www.feednetback.eu).
}

In almost all cases, the suboptimality of the resulting control law (and as a consequence the stability of the feedback system) is valid under various strong assumptions. Examples include the approximation of the Lipschitz constant (Canale et al. [2009a]) and the availability of global optimization tools (Johansen [2004]). While these approaches indeed work well in practice, in many problems the stability of the closed-loop system (and the resulting region of attraction) can not be guaranteed. Thus, in this paper we exploit advances in reachability analysis and adaptive interpolation to construct an approximate explicit control law that encompasses the strengths of the recent works (Canale et al. [2009a], Johansen [2004]), while guaranteeing stability and feasibility and preserving a minimal representation of the control law.

Extending the results of Summers et al. [2009], in this paper we introduce a constructive algorithm for the approximation of an explicit receding horizon NMPC control law. We approximate the optimal control law by adaptive interpolation using second order interpolets, while concurrently verifying feasibility and stability of the resulting feedback system via the computation of an inner approximation of the capture basin (see e.g. Delanoue et al. [2008]). With the approach we propose, we are able to construct a sparse approximation of the optimal control law while taking into consideration performance loss and the feasibility and stability of the feedback system. Further, since the solution is defined on a gridded hierarchy, the online evaluation of the control law is extremely fast Summers et al. [2009].

The rest of the paper is arranged as follows. In section 2 we introduce the NMPC problem. In section 3 we construct a d-dimension multiscale basis function and introduce an adaptive thresholding approach for sparse function approximation. In section 4 we introduce the notions of reachability analysis and address how to compute a basin of attraction using interval methods. In section 5 we 
outline the algorithm for the construction of the explicit NMPC control law. In section 6 we provide a numerical example of the method.

\section{NONLINEAR MODEL PREDICTIVE CONTROL}

The recent interest in parametric programming in the control community has arisen from the ability to pose certain optimal control problems as parametric programs, resulting in numerous methods to pre-compute the optimal control law offline. In this paper we are specifically interested in the following finite horizon optimal control problem (NMPC):

$$
\begin{array}{r}
J^{*}(x)=\quad \min _{\left\{u_{0}, \ldots, u_{N-1}\right\}} J\left(u_{0}, \ldots, u_{N-1}, x_{0}, \ldots, x_{N}\right) \\
\text { s.t. } \quad x_{i+1}=f\left(x_{i}, u_{i}\right), \forall i=0, \ldots, N-1 \\
\left(x_{i}, u_{i}\right) \in \mathcal{X} \times \mathcal{U}, \forall i=0, \ldots, N-1 \\
x_{N} \in \mathcal{X}_{F}, \\
x_{0}=x,
\end{array}
$$

where $x_{i} \in \mathbb{R}^{n}$ is the state of the system, $u_{i} \in \mathbb{R}^{m}$ is the control input of the system, and $N$ is the prediction horizon length. In the current formulation, we assume that the cost function takes the form

$$
J\left(u_{0}, \ldots, u_{N-1}, x_{0}, \ldots, x_{N}\right):=V_{N}\left(x_{N}\right)+\sum_{i=0}^{N-1} L\left(x_{i}, u_{i}\right),
$$

where $L$ is the running (stage) cost and $V_{N}$ is the terminal cost. We also assume that $f: \mathbb{R}^{n} \times \mathbb{R}^{m} \rightarrow \mathbb{R}^{n}$ is a continuous function and that the objective is to regulate the state of the system to the origin under state, control input, and terminal state constraints represented by the (compact) sets $\mathcal{X} \subseteq \mathbb{R}^{n}, \mathcal{U} \subseteq \mathbb{R}^{m}$, and $\mathcal{X}_{F} \subseteq \mathbb{R}^{m}$. For the sake of simplicity (as in Canale et al. [2009a], Johansen [2004]), we assume that the control input constraint set $\mathcal{U}$ is convex, although the following results can be extended to the nonconvex control input constraint setting.

Problem (1) can be re-written as a parametric optimization problem:

$$
u^{*}(x):=\arg \min _{u}\{h(x, u) \mid g(x, u) \leq 0\},
$$

where $u$ is a vector containing the sequence of control inputs $u_{0}, \ldots, u_{N-1}$ and appropriate auxiliary variables and the functions $h$ and $g$ are generally nonconvex. We assume that the terminal set $\mathcal{X}_{F}$ is positively control invariant under a known stabilizing feedback law. The system input is then applied in a receding horizon fashion (Mayne et al. [2000]) by $u_{0}^{*}(x)$, which is the first input in the optimal control sequence of (1). A dual mode approach is adopted, i.e. once the terminal set is attained, the stabilizing feedback law defined in $\mathcal{X}_{F}$ is applied. Following (Canale et al. [2009a], Johansen [2004]), we assume in this paper that the optimal receding horizon control law $u_{0}^{*}(x)$ asymptotically stabilizes the origin of the closedloop system.

\section{MULTISCALE FUNCTION APPROXIMATION}

Our method of approximating (3) effectively relies on coarsely gridding the state space, and then systematically regridding with increasing resolution the regions which have not been approximated sufficiently (i.e. not all grid points are evaluated) while only keeping the grid points which play a significant role in the function approximation. We start with a brief introduction of the sparse multiresolution methodology for function approximation (Summers et al. [2009]). The reader is referred to DeVore [1998], Mallat [1989], Bungartz and Griebel [2004] for more information on which this section is based.

Define the standard one-dimensional scaling function (hat function) with support $[-1,1]$ by

$$
\phi(x):= \begin{cases}1-|x|, & \text { if } x \in[-1,1] \\ 0, & \text { otherwise. }\end{cases}
$$

In one dimension, we consider a dyadic discretization on the unit interval $\Omega=[0,1]$. The resulting grid $\Omega_{l}$ is characterized by the level of discretization $l$ and the index $i$. At level $l$ the distance between points is $h_{l}=2^{-l}$ and the number of points is $N=2^{l}+1$. The index $i$ determines the location of the grid points according to the equation

$$
x_{l, i}:=i \cdot h_{l}, \quad 0 \leq i \leq 2^{l} .
$$

Consider $\phi_{l, i}$ a family of basis functions defined on $\Omega$ with support $\left[x_{l, i}-h_{l}, x_{l, i}+h_{l}\right]$. The function $\phi_{l, i}$ is generated from function (4) via translation and dilation,

$$
\phi_{l, i}(x)=\phi\left(\frac{x-i \cdot h_{l}}{h_{l}}\right) .
$$

The family of univariate multiscale functions $\psi_{l, i}$ which make up the hierarchical basis is given as

$$
\psi_{l, i}=\phi_{l, i}, i \in I_{l} .
$$

where

$$
I_{l}=\left\{\begin{array}{ll}
\left\{i \in \mathbb{N}_{0} \mid 1 \leq i \leq 2^{l}-1, i \text { odd }\right\} & l>l_{0} \\
\left\{i \in \mathbb{N}_{0} \mid 0 \leq i \leq 2^{l}\right\} & l=l_{0}
\end{array} .\right.
$$

Let $\mathbf{y} \in \mathbb{N}_{0}^{d}$ denote a $d$-dimensional multi-index (i.e. $\mathbf{y}$ is a d-vector of indices taking values in the nonnegative integers), where operations (e.g. addition) and comparisons (e.g. $\leq$ ) hold component-wise. A multivariate multiscale basis on the unit cube $\Omega^{d}=[0,1]^{d}$, where $d$ is the dimension, can be constructed by tensor product expansion of the one-dimensional multivariate functions $\psi_{l, i}$ (see Figure 1), i.e.

$$
\psi_{l, \mathbf{i}}=\prod_{j=1}^{d} \psi_{\left(l, i_{j}\right)}
$$

with the multi-index $\mathbf{i} \in I_{l}^{d}$ and

$$
I_{l}^{d}=\left\{\begin{array}{l}
\left\{\mathbf{i} \in \mathbb{N}_{0}^{d} \mid \mathbf{0} \leq \mathbf{i} \leq \mathbf{2}^{l}\right\} \backslash \\
\left\{\mathbf{i} \in \mathbb{N}_{0}^{d} \mid \mathbf{0} \leq \mathbf{i} \leq \mathbf{2}^{l}, i_{j} \text { even } \forall j \in[1, d]\right\} \\
\left\{\mathbf{i} \in \mathbb{N}_{0}^{d} \mid \mathbf{0} \leq \mathbf{i} \leq \mathbf{2}^{l}\right\} \\
l>l_{0}, \\
l=l_{0} .
\end{array}\right.
$$

Note that $I_{l}^{d}$ is simply the full grid less those points seen at previous levels, as depicted in Figure 2. We may now define the d-dimensional hierarchical function spaces of piecewise d-linear functions as $W_{l}^{d}=\operatorname{span}\left\{\psi_{l, \mathbf{i}}: \mathbf{i} \in I_{l}^{d}\right\}$. Defining the family of d-dimensional nodal basis functions 


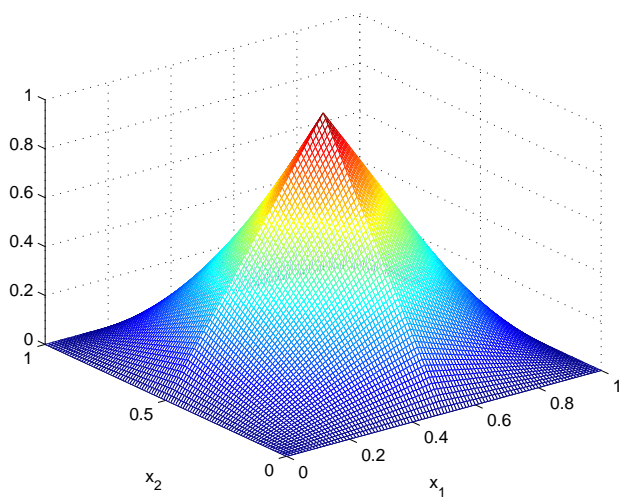

Fig. 1. Two dimensional hierarchical basis function by tensor product expansion of the one dimensional hat function.

$$
\phi_{l, \mathbf{i}}(x)=\prod_{j=1}^{d} \phi_{l, i_{j}}\left(x_{j}\right)
$$

and the d-dimensional nodal function space $V_{l}^{d}=$ $\operatorname{span}\left\{\phi_{l, \mathbf{i}}: \mathbf{0} \leq \mathbf{i} \leq \mathbf{2}^{l}\right\}$, it can be shown that $V_{l}^{d}=$ $\bigoplus_{k \leq l} W_{k}^{d}$ where $\bigoplus$ denotes the direct sum.

Any function $u_{l} \in V_{l}^{d}$ can be uniquely represented in the nodal basis as a weighted sum of basis functions

$$
u_{l}(x)=\sum_{\mathbf{i}=\mathbf{0}}^{\mathbf{2}^{l}} v_{l, \mathbf{i}} \cdot \phi_{l, \mathbf{i}}(x)
$$

where the coefficients $v_{l, \mathbf{i}}$ are equal to the function value $u_{l}\left(x_{l, \mathbf{i}}\right)$. Likewise, the function $u_{l} \in V_{l}^{d}$ can also be uniquely represented in the hierarchical basis by

$$
u_{l}(x)=\sum_{k=\mathbf{0}}^{l} \sum_{\mathbf{i} \in I_{k}^{d}} w_{k, \mathbf{i}} \cdot \psi_{\mathbf{k}, \mathbf{i}}(x)
$$

with coefficients $w_{k, \mathbf{i}} \in \mathbb{R}$, commonly referred to as the hierarchical details, corresponding to the difference between the true function value $u_{l}\left(x_{k, \mathbf{i}}\right)$ and the value of the approximate function one level below.

A common thresholding argument, and the one we will employ throughout this paper, $\left|w_{k, \mathbf{i}}\right| \geq \delta$, simply reduces to taking the absolute value of the detail coefficient greater than the tolerance, and discarding the weight $w_{k, \mathbf{i}}$ if it is lower than the threshold. In this manner, an adaptive function approximation can be expressed

$$
\hat{u}(x)=\sum_{(k, \mathbf{i}) \in \Lambda^{\delta}(u)} w_{k, \mathbf{i}} \cdot \psi_{k, \mathbf{i}}(x),
$$

where the 'active' index set $\Lambda^{\delta}(u)$ is the index set corresponding to all detail coefficients larger than $\delta$.

\section{FEASIBILITY AND STABILITY}

In Summers et al. [2009], it was shown that the approximate control law built from the adaptive multiscale basis functions can be separated into hypercubic regions spanned by an interpolation by barycentric coordinates.

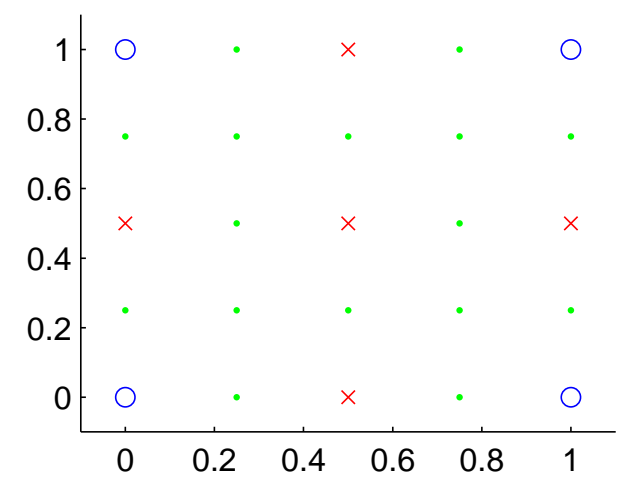

Fig. 2. Grid points for subspaces $W_{0}^{2}$ (circles), $W_{1}^{2}$ (x's), and $W_{2}^{2}$ (dots).

We denote the set of all such hypercubic regions up to maximum resolution $l$ as $\bar{R}$.

Consider a hierarchical approximate control law defined on $\Omega^{d} \subset \mathbb{R}^{d}\left(\mathcal{X}_{F} \subseteq \mathcal{X} \subseteq \Omega^{d}\right)$ with maximum level $l$

$$
\hat{u}(x)=\sum_{(k, \mathbf{i}) \in \Lambda^{\delta}\left(u^{*}\right)} w_{k, \mathbf{i}} \cdot \psi_{k, \mathbf{i}}(x)
$$

where $u^{*}$ is given by (3). By Summers et al. [2009], we see that the approximate control law defined on $R \in \bar{R}$ can be expressed as an interpolation by barycentric coordinates

$$
\hat{u}(x)=\sum_{v \in \operatorname{extr}(R)} \hat{u}(v) f_{v}(x), \text { if } x \in R
$$

where $\operatorname{extr}(R)$ are the extreme points of $R$ and $f_{v}(x)$ are compactly supported basis functions of the form (6) centered at the corners of the hypercube $R$.

\subsection{Capture basin}

Consider the discrete time system $x_{i+1}=f\left(x_{i}, u_{i}\right)$ with the control law (7). We can rewrite such a closed loop system as

$$
x_{i+1}=\bar{f}_{R}\left(x_{i}\right), \forall x \in R, \quad R \in \bar{R} .
$$

Given the autonomous system (9), define the capture basin Aubin [1991], Saint-Pierre [1994] as the set of initial states $R_{s} \subseteq \mathcal{X}$ for which the terminal invariant set $\mathcal{X}_{F}$ is attainable in finite time while satisfying at the same time the system constraints. Under the assumption that the terminal set is invariant and has a predefined control law guaranteeing asymptotic stability, such a set is the region of attraction of the origin for system (9).

In general, the capture basin cannot be explicitly computed. However, we may use the following procedure to compute an inner approximation of the capture basin.

Note that state and control constraints are satisfied in $R_{s}$. In fact, the control laws inside each hypercube $R$ satisfy the control constraints (they are obtained as an interpolation of feasible control laws at the vertices of the boxes). Moreover, state constraints are also satisfied by requiring that $\bar{R} \subseteq \mathcal{X}$.

As described in the procedure, outer approximations of the reachable sets will need to be computed. In fact, as 


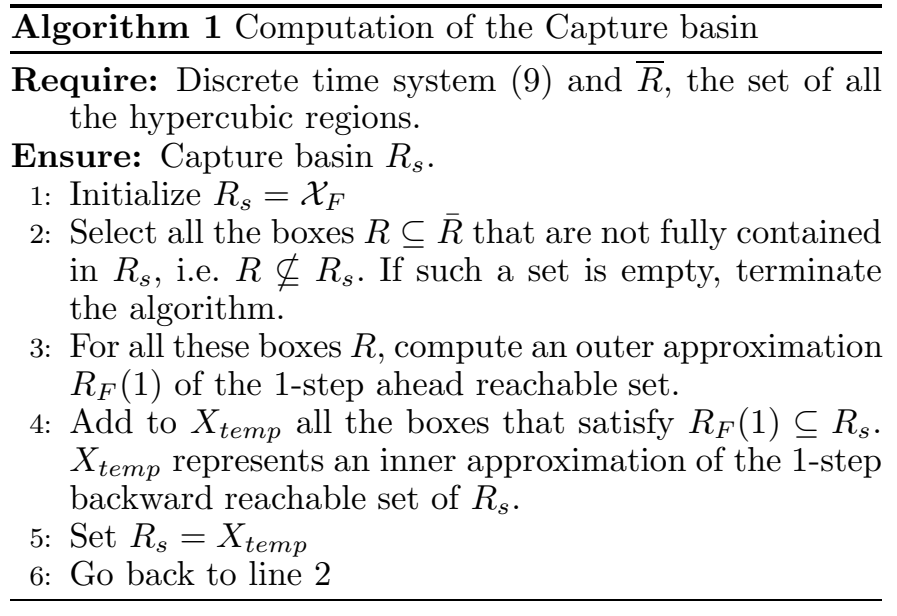

discussed in Kuhn [1998], it is not possible to find exactly the set $\Phi$,

$$
\Phi=\bar{f}_{R}(R),
$$

given an initial set $R \in \mathbb{R}^{n}$ and a generic nonlinear map $\bar{f}_{R}$. However, an overestimation of $\bar{f}_{R}(R)$ can be obtained. The objective is hence to compute an outer approximation of the reachable sets of (10) in such a way that the overestimation is kept small. Several methods have been proposed in literature. In the following, intervals are recalled (see Moore [1966] for more details). Alternate methods, e.g. zonotopes, can reduce the conservativeness of intervals at the expense of computational burden. The interested reader can refer to the following papers Kuhn [1998] and Combastel [2005]. For sake of space we will explore just the interval analysis approach.

\subsection{Interval Arithmetic}

Given $a, b \in \mathbb{R}$, with $a \leq b$, a scalar interval $H=[a, b]$ is the set $\{h: a \leq h \leq b\}$, i.e. a closed and connected subset of $\mathbb{R}$. The unit interval is denoted by $\mathbf{B}=[-1,1]$. I denotes the set of all real intervals of $\mathbb{R}$. Denoting with $\bullet$ any one of $(+,-, \times, \div)$, basic operations on scalar intervals $A, B \in \mathbb{I}$ satisfy the following

$$
A \bullet B=\{a \bullet b: a \in A, b \in B\} .
$$

Following this procedure, the four basic interval operations are defined in Moore [1966]. An interval vector of $\mathbb{R}^{n}$ can be viewed as a box. $\mathbb{I}^{n}$ denotes the set of all boxes of $\mathbb{R}^{n}$. A unitary box $\mathbf{B}^{n}$, is a box composed by $n$ unitary intervals. The interval extension of standard functions (sin, cos, tan, arctan, exp, ln, abs, sqrt) as well as matrix operations with intervals are also possible (see Moore [1966]). In this work, the INTLAB interval toolbox Rump [1999] has been used.

Definition 1. (Natural interval extension Kearfott [1996]) If $f: \mathbb{R}^{n} \rightarrow \mathbb{R}^{n}$ is a function computable as an expression, algorithm or computer program involving the four elementary arithmetic operations interspersed with evaluations of standard functions then, a natural interval extension of $f$, denoted $\square f$, is obtained replacing each occurrence of each variable by the corresponding interval variable, by executing all operations according to interval operators and by computing ranges of the standard functions.
Theorem 11. Moore [1966] A natural interval extension $\square f$ of a continuous function $f: \mathbb{R}^{n} \rightarrow \mathbb{R}^{n}$ over a box $H \subseteq \mathbb{R}^{n}$ satisfies $f(H) \subseteq \square f(H)$.

Theorem 12. Kearfott [1996] A natural interval extension $\square f$ of a continuous function $f: \mathbb{R}^{n} \rightarrow \mathbb{R}^{n}$ over two boxes $H, Y \subseteq \mathbb{R}^{n}$ such that $H \subseteq Y$ satisfies that $\square f(H) \subseteq$ $\square f(Y)$.

Figure 3 illustrates an example of application of the natural interval extension.

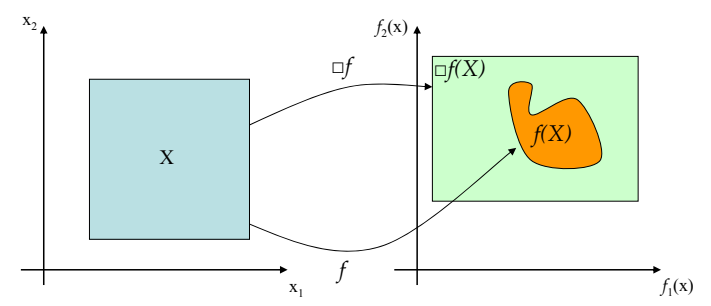

Fig. 3. Illustration of natural interval extension

\section{APPROXIMATE EXPLICIT NMPC}

We now introduce a recursive algorithm for the multiresolution approximation of the explicit nonlinear model predictive control law. The algorithm initializes at a user defined coarse uniform grid, and then proceeds with a dyadic refinement strategy, saving only the points which violate a user defined thresholding law and/or feasibility condition. Exploiting the fact that the state space can be decomposed into a union of hypercubes (with respect to the approximate receding horizon control law), the algorithm restricts the dyadic refinement to the hypercubes intersecting the current invariant set, thus constructing a basin of attraction from the inside out (starting from the terminal set) by iteration.

Pseudocode for the approximation process is given in Algorithm 2. For state $x \in \mathbb{R}^{d}$ and control $u \in \mathbb{R}^{m}$, the model predictive control problem is defined as in (1) through the choice of system dynamics $f(x, u)$, state and control constraints $\mathcal{X}$ and $\mathcal{U}$, terminal set constraint $\mathcal{X}_{F}$, time horizon $N$, and cost function (2). The overall performance index (which can only be enforced pointwise) defined by the user is given by the threshold coefficient $\epsilon \in[0,1]$ such that only coefficients which result in a cost function error larger than $\epsilon L(x, 0)$ are kept (where $L$ is the stage cost of the NMPC problem). Note that this argument not only controls the pointwise performance loss of the approximate control law, but additionally guarantees stability and feasibility of the pointwise receding horizon control law (see Summers et al. [2009]). The index set $\Lambda$ is initialized at level $l_{0}$ along with all indices and details. As the grid is refined, $\Lambda$ stores the levels of resolution $k$ and indices corresponding to the set of hierarchical details that are not discarded due to falling below the required approximation level. The maximum level of resolution is given as $l_{\max }$. The set of stored detail coefficients is given by the set $\mathbf{w}$. The region of attraction is given by the set $R_{s}$ and is initialized to the terminal set $R_{s}=\mathcal{X}_{F}$. Additionally, the set of hypercubes intersecting the region of attraction (capture basin) is denoted $R_{c}$ and 


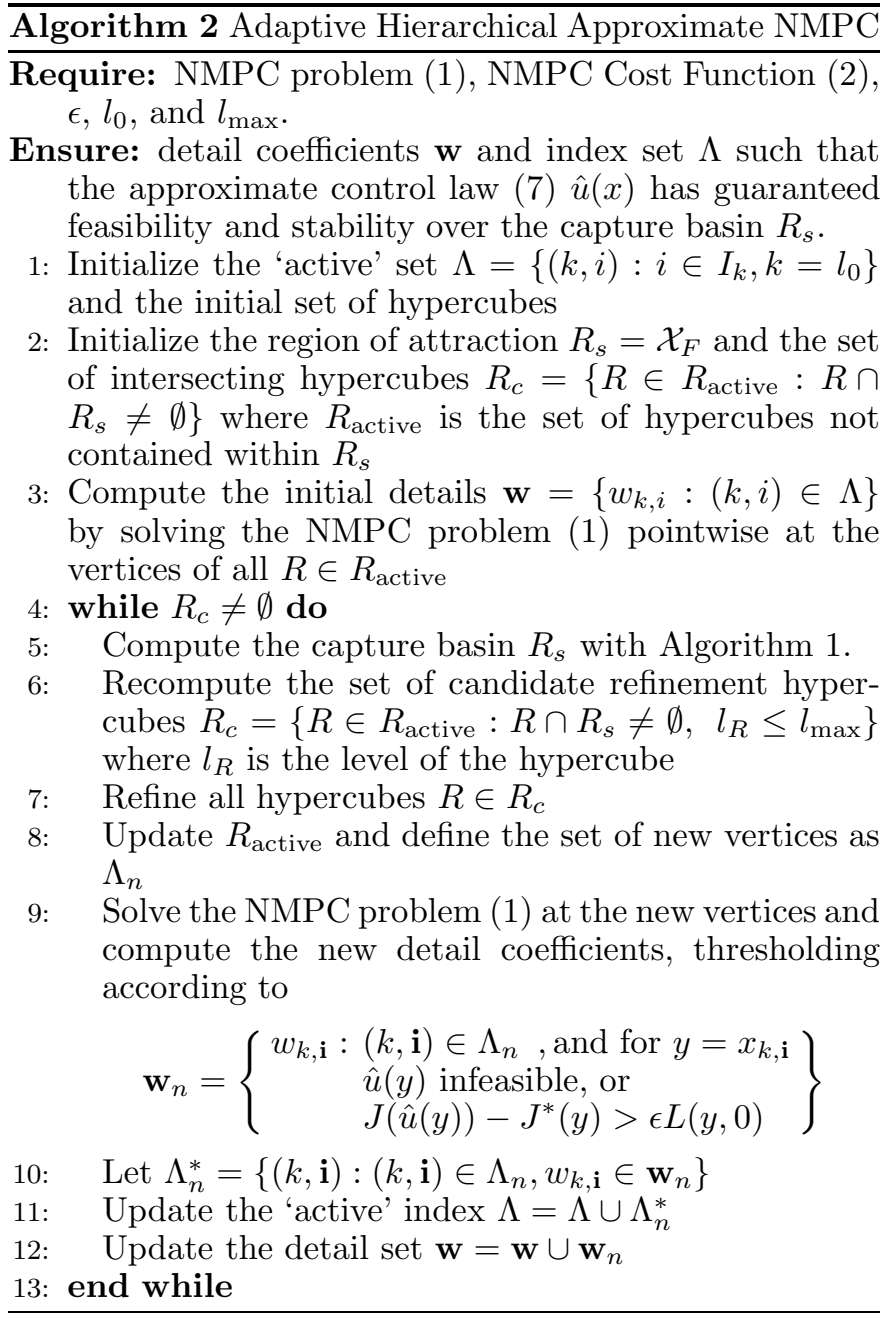

represents the set of refinement candidate sets. Lastly, $R_{c} \subseteq R_{\text {active }}$ where $R_{\text {active }}$ is the set of hypercubes not contained within $R_{s}$. Note that the complexity of the realtime implementation of the approximate control law can be found in Summers et al. [2009].

We now introduce the main theorem which proves that Algorithm 2 will always provide a stabilizing receding horizon control law and verifiable region of attraction for the NMPC problem (1), and further that the function approximation can approximate the true control law to arbitrary precision.

Theorem 13. Let $\hat{u}_{0}$ be the resulting receding horizon approximate control law computed from Algorithm 2 for the NMPC problem (1) with cost $(2), \epsilon \in[0,1], l_{0} \in \mathbb{N}$, and $l_{\max } \in \mathbb{N}$. The following properties hold for $\hat{u}_{0}$ :

a) Asymptotic stability to the origin for all $x_{0} \in R_{s}$

b) $\hat{u}_{0} \in \mathcal{U}$ for all $x \in R_{s}$

c) For all $x_{0} \in R_{s}, x_{i} \in \mathcal{X}$ for all $i=1,2,3, \ldots$

d) $R_{s} \supseteq \mathcal{X}_{F}$

e) As $\epsilon \rightarrow 0$ and $l_{\max } \rightarrow \infty$, then $\hat{u}_{0} \rightarrow u_{0}^{*}$ and $R_{s} \rightarrow \mathcal{R}$ where $\mathcal{R}$ is the maximum invariant set for (1)

Proof. The first item holds since the computation of $R_{s}$ by Algorithm 1, coupled with the assumption that the terminal set $\mathcal{X}_{F}$ is positively invariant with respect to a known feedback law, implicitly defines a stabilizing Lyapunov function with respect to the dual mode receding horizon approximate control law $\hat{u}_{0}$. Feasibility of the control law holds as a result of the interpolation by barycentric coordinates and the assumption that $\mathcal{U}$ is convex (as stated in Section 2). State constraint satisfaction of all trajectories whose initial state lies in the capture basin $R_{s}$ is guaranteed by the definition of the capture basin. The fourth item is trivial according to the initialization of Algorithm 2. The final item holds since the second order interpolets form a basis for the space of continuous and compactly supported functions. This implies that, given enough detail coefficients (i.e. if $l_{\max }$ is large enough), $\hat{u}_{0}$ can approximate $u_{0}^{*}$ to any precision (i.e. any $\epsilon \in[0,1]$ ).

\section{NUMERICAL EXAMPLE}

Consider the following two-dimensional continuous-time nonlinear system (e.g. see Chen and Allgower [1998], Johansen [2004], Canale et al. [2009b])

$$
\begin{aligned}
& \dot{x}_{1}(t)=x_{2}(t)+\left[\mu+(1-\mu) x_{1}(t)\right] u(t) \\
& \dot{x}_{2}(t)=x_{1}(t)+\left[\mu-4(1-\mu) x_{2}(t)\right] u(t)
\end{aligned}
$$

with $\mu=0.5$. It is well known (Chen and Allgower [1998]) that the origin of the system governed by (14) and (15) is unstable, and that the linearized system is stabilizeable (but not controllable) for any $\mu \in(0,1)$.

In consideration of the NMPC problem (1), the system (14) and (15) is discretized using a forward difference Euler approximation with sampling time $T=0.1$. The input and state constraint sets are $\mathcal{U}=\{u \in \mathbb{R}:|u| \leq 2\}$ and $\mathcal{X}=\left\{x \in \mathbb{R}^{2}:\|x\|_{\infty} \leq 1\right\}$. The cost function is defined over a prediction horizon of length $N=15$ as

$$
\begin{aligned}
J\left(u_{0}, \ldots, u_{N-1}, x_{0}, \ldots, x_{N}\right):= & x_{N}^{T} P x_{N} \\
& +\sum_{i=0}^{N-1} x_{i}^{T} Q x_{i}+u_{i}^{T} R u_{i},
\end{aligned}
$$

where

$$
Q=\left[\begin{array}{ll}
0.05 & 0 \\
0 & 0.05
\end{array}\right], \quad R=0.1, \quad P=\left[\begin{array}{ll}
16.5926 & 11.5926 \\
11.5926 & 16.5926
\end{array}\right] .
$$

The terminal penalty matrix $P$ is given by the solution to the Lyapunov-equation providing a positively invariant terminal set $\mathcal{X}_{F}=\left\{x \in \mathbb{R}^{2}: x^{T} P x \leq 0.7\right\}$ (see Chen and Allgower [1998]).

With $\epsilon=0.5, l_{0}=1$, and $l_{\max }=7$, we compute a stabilizing control law using Algorithm 2 that consists of 581 hierarchical details spanning 7 levels. All computations were performed in MatLab. The function fmincon was used to solve the pointwise NMPC problem (1) and the INTLAB interval toolbox Rump [1999] was used to recursively compute the capture basin. In Figure 4 , the resulting approximate receding horizon control law and approximate (inner) control invariant set are shown. Additionally, 1000 Monte Carlo simulations were completed for a horizon length $N_{m}=20$ with the initial states drawn randomly from the feasible region according to a uniform distribution. The results included an average closed loop cost increase of less than 0.2 percent.

Assuming a processor speed of 1 Gflops/s, the explicit multiscale control law for this two-dimensional example can be evaluated in $31 \mathrm{~ns}$ or $0.5 \mu \mathrm{s}$ according to the structures introduced in Summers et al. [2009]. 


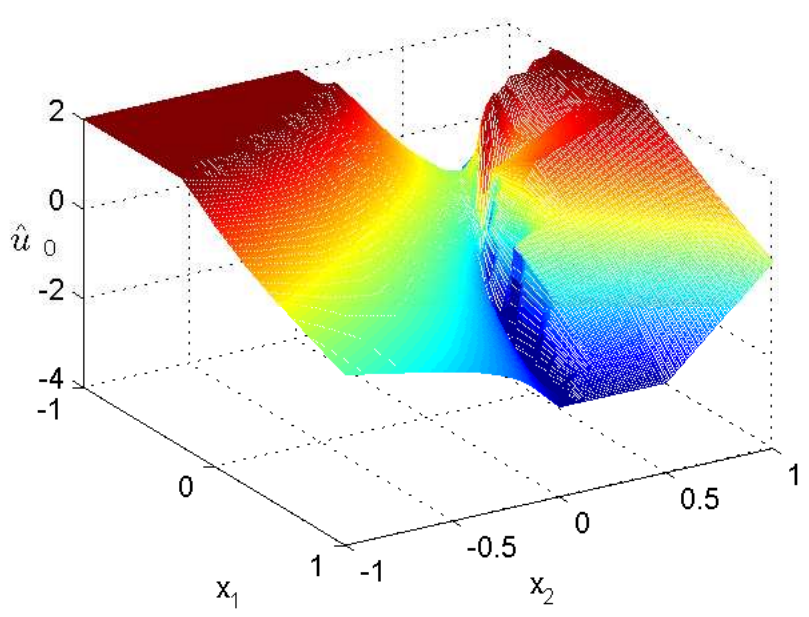

(a) Approximate control law

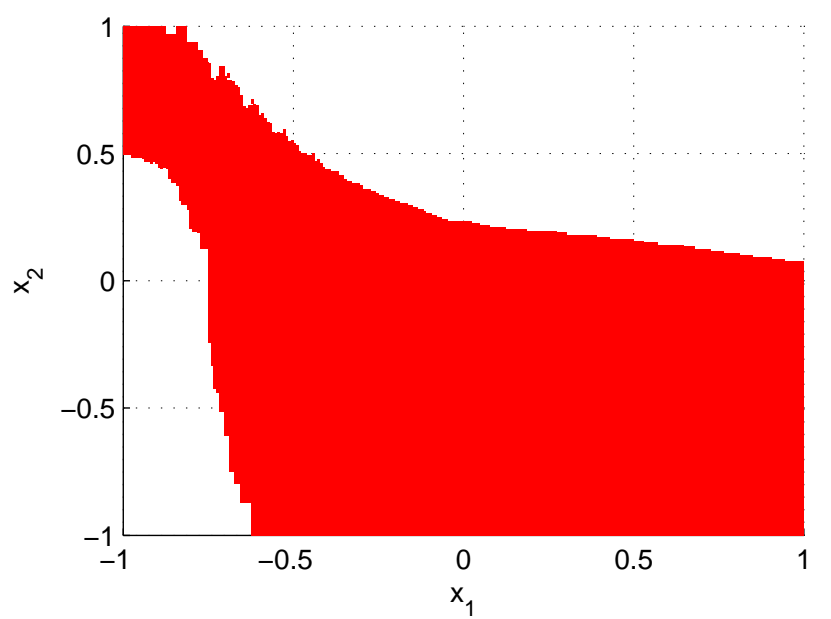

(b) Feasible Region

Fig. 4. The approximate receding horizon control law $\hat{u}_{0}$ is shown in (a) and the feasible and stable region is depicted in $(b)$.

\section{REFERENCES}

Jean-Pierre Aubin. Viability Theory. Birkhauser Boston Inc., Cambridge, MA, USA, 1991. ISBN 0-8176-3571-8.

A. Bemporad, M. Morari, V. Dua, and E. N. Pistikopoulos. The explicit linear quadratic regulator for constrained systems. Automatica, 38(1):3-20, January 2002.

Hans-Joachim Bungartz and Michael Griebel. Sparse grids. Acta Numerica, 13:147-269, 2004.

M. Canale, L. Fagiano, and M. Milanese. Set membership approximation theory for fast implementation of model predictive control laws. Automatica, 45(1), 2009a.

M. Canale, L. Fagiano, M. Milanese, and C. Novara. Set Membership Approximations of Predictive Control Laws: The Tradeoff between Accuracy and Complexity. In European Control Conference, Budapest, Hungary, August 2009b.

H. Chen and F. Allgower. A quasi-infinite horizon nonlinear model predictive control scheme with guaranteed stability. Automatica, 34(10):1205-1218, 1998.

C. Combastel. A state bounding observer for uncertain non-linear continuous-time systems based on zonotopes. In IEEE Conference on Decision and Control, volume 44, page 7228. IEEE; 1998, 2005.

N. Delanoue, L. Jaulin, L. Hardouin, and M. Lhommeau. Guaranteed Characterization of Capture Basins of Nonlinear State-Space Systems. In Informatics in Control, Automation and Robotics: Selected Papers from the International Conference on Informatics in Control, Automation and Robotics 200\%, page 265. Springer, 2008.

Ronald A. DeVore. Nonlinear approximation. Acta Numerica, 7(-1):51-150, 1998.

T. A. Johansen. Approximate explicit receding horizon control of constrained nonlinear systems. Automatica, 40(2):293-300, 2004.

Tor A. Johansen and Idar Petersen. On explicit suboptimal LQR with state and input constraints. In in Proc. IEEE Conf. Decision and Control, pages 05-6, 2000.

R.B. Kearfott. Rigorous global search: continuous problems. Kluwer Academic Pub, 1996.

W. Kuhn. Rigorously computed orbits of dynamical systems without the wrapping effect. Computing, 61
(1):47-67, 1998.

G. C. Goodwin M. M. Seron and J. A. De Dona. Geometry of model predictive control including bounded and stochastic disturbances under state and input constraints. Technical report, University of Newcastle, 2000 .

L. Magni, D. M. Raimondo, and F. Allgöwer, editors. Nonlinear Model Predictive Control: Towards New Challenging Applications. Springer-Verlag, 2009.

Stephane G. Mallat. A theory for multiresolution signal decomposition: the wavelet representation. IEEE Transactions on Pattern Analysis and Machine Intelligence, 11:674-693, 1989.

D. Q. Mayne, J. B. Rawlings, C. V. Rao, and P. O. M. Scokaert. Constrained model predictive control: Stability and optimality. Automatica, 36(6):789-814, June 2000.

RE Moore. Interval analysis (Prentice-Hall Series in Automatic Computation). 1966.

Manfred Morari and Jay H. Lee. Model predictive control: past, present and future. Computers \& Chemical Engineering, 23(4-5):667 - 682, 1999.

G. Pin, M. Filippo, A. Pellegrino, and T. Parisini. Approximate off-line receding horizon control of constrained nonlinear discrete-time systems. In Proc. European Control Conference, pages 2420-2431, Budapest, Hungary, August 2009.

J. B. Rawlings and D. Q. Mayne. Model Predictive Control: Theory and Design. Nob Hill Publishing, 2009.

S.M. Rump. INTLAB - INTerval LABoratory. In Tibor Csendes, editor, Developments in Reliable Computing, pages 77-104. Kluwer Academic Publishers, Dordrecht, 1999.

Patrick Saint-Pierre. Approximation of the viability kernel. Applied Mathematics and Optimization, 29(2):187109, 1994.

S. Summers, C.N. Jones, J. Lygeros, and M. Morari. A Multiscale Approximation Scheme for Explicit Model Predictive Control with Stability, Feasibility, and Performance Guarantees. In IEEE Conference on Decision and Control, Shanghai, China, December 2009. 